\title{
CONFORMALLY FLAT IMMERSIONS AND FLATNESS OF THE NORMAL CONNECTION
}

\author{
Maria Helena Noronha
}

\begin{abstract}
B. Y. Chen and T. Teng affirmed that almost umbilic isometric immersions of an $n$-dimensional manifold in $\mathbf{R}^{n+2}$ have zero normal curvature $\left(R^{\perp}=0\right)$. In this paper we exhibit a counterexample to this statement and we prove that either $R^{\perp}=0$ or there exists (locally) an isometric immersion of this manifold in $\mathbf{R}^{n+2}$ with $R^{\perp}=0$. These immersions are conformally flat and we study their local geometry.
\end{abstract}

1. Introduction. An $n$-dimensional Riemannian manifold $M^{n}$ is conformally flat if, for each $x \in M$, there exists a conformal diffeomorphism of a neighborhood of $x$ onto an open set of the Euclidean space $\mathbf{R}^{n}$. We will call conformally flat immersion (CFI for short) an immersion $f: M^{n} \rightarrow \mathbf{R}^{n+p}$ with $M$ conformally flat in the metric induced by $f$.

A nice characterization of CFI in terms of the second fundamental form was given by Moore in [4] and Moore-Morvan in [5].

In this paper we want to consider CFI with codimension two. From [5], we can conclude that if $n \geq 5$, such an isometric immersion $f$ is almost umbilic, i.e., for every $x \in M$ there exists an orthonormal frame of the normal space $T_{x} M^{\perp}$ such that for each $\xi$ of this frame, the Weingarten operator $A_{\xi}$ has an eigenvalue of multiplicity at least $n-1$.

In [2] there exists a false statement which says that "almost umbilic isometric immersions in Euclidean space with codimension two have zero normal curvature". This and [5] together would imply that CFI in codimension two has zero normal curvature $\left(R^{\perp}=0\right)$. In $\S 2$ we will discuss this false result and its counterexample. The aim of this paper is to show that if a conformally flat manifold can be isometrically immersed in $\mathbf{R}^{n+2}$ then either $R^{\perp}=0$ or there is a local isometric immersion in $\mathbf{R}^{n+2}$ with $R^{\perp}=0$.

Before stating our results, we will recall some definitions below:

Definition. A submanifold $\Sigma \subset M$ is a geometric sphere of type $\varepsilon$, $\varepsilon=0,1$, if $\Sigma$ is an umbilic submanifold with parallel mean curvature vector and such that the sectional curvature of $M$ along planes tangent 
to $\Sigma$ are constant $k, k=0$ if $\varepsilon=0, k>0$ if $\varepsilon=1$. If a conformally flat manifold $M^{n}$ is locally foliated by geometric spheres of type $\varepsilon$ and codimension $s$, we will say that $M^{n}$ is of type $(s, \varepsilon)$.

In the case of CFI with codimension two, it follows from [4] and [7] that the connected components of an open dense $V \subset M$ are either umbilic, of type $(1, \varepsilon)$ or of type $(2, \varepsilon)$. It will be easy to prove that a CFI of manifold of type $(1, \varepsilon)$ with $\varepsilon=1$ has $R^{\perp}=0$. Thus, our main result is:

THEOREM. Let $f: M^{n} \rightarrow \mathbf{R}^{n+2}$ be a CFI where $M$ is of type $(2, \varepsilon)$ and $n \geq 5$. Then $M$ is (locally) foliated by two orthogonal codimension one foliations whose leaves are, in the induced metric, conformally flat manifolds of type $(1, \varepsilon)$ and the intersection of the two foliations gives the codimension two foliation by geometric spheres. Conversely, if $M$ admits such foliations, $M$ can be (locally) isometrically immersed in $\mathbf{R}^{n+2}$ with $R^{\perp}=0$.

The author wants to thank Professor F. Mercuri for bringing our attention to the example (2.1) below.

2. Flat $n$-dimensional manifold in $\mathbf{R}^{n+2}$ with $R^{\perp} \neq 0$.

(2.1) EXAMPLE. Let $f: M^{2} \rightarrow \mathbf{R}^{4}$ be an isometric immersion with $R^{\perp} \neq 0$ and $M^{2}$ flat (this immersion can be the composition of a cylindrical immersion of $\mathbf{R}^{2}$ into $\mathbf{R}^{3}$ with another cylindrical immersion of $\mathbf{R}^{3}$ into $\mathbf{R}^{4}$ along a curve whose tangent direction is not perpendicular or equal to the principal direction of the first immersion). Consider the product immersion $\bar{f}: M^{2} \times \mathbf{R}^{n-2} \rightarrow \mathbf{R}^{n+2}$. We see that $\bar{f}$ satisfies $R^{\perp} \neq 0$ and that $M^{2} \times \mathbf{R}^{n-2}$ is flat, in particular, is conformally flat.

(2.2) Assertion. If $n \geq 5$, the example (2.1) is an almost umbilic immersion.

In fact, let $\left(\xi_{1}, \xi_{2}\right)$ be an orthonormal frame of $T_{x} M^{\perp}$ and let $\left(X_{1}, \ldots, X_{n}\right)$ be an orthonormal frame in $T_{x} M$ which diagonalizes the Weingarten operator $A_{\xi_{1}}$. Setting $\xi_{\theta}=\cos \theta \xi_{1}+\sin \theta \xi_{2}$, we claim that there exists $\theta$ such that $\xi_{\theta}$ is almost umbilic. It is enough to take $\theta$ as a solution of the following equation:

$$
\lambda_{1} \lambda_{2} \cos ^{2} \theta+\left(a \lambda_{2}+c \lambda_{1}\right) \cos \theta \sin \theta+\left(a b-c^{2}\right) \sin ^{2} \theta
$$

where

$$
A_{\xi_{1} \mid T M^{2}}=\left[\begin{array}{cc}
\lambda_{1} & 0 \\
0 & \lambda_{2}
\end{array}\right], \quad A_{\xi_{2} \mid T M^{2}}=\left[\begin{array}{ll}
a & c \\
c & b
\end{array}\right] .
$$


We observe that the above equation has a solution because $\lambda_{1} \lambda_{2}+$ $\left(a b-c^{2}\right)=0$. The lemma below allows us to find the other almost umbilic direction.

(2.3) Lemma. Let $f: M^{n} \rightarrow \mathbf{R}^{n+2}$ be a CFI, $n \geq 5$ and $\xi$ an almost umbilic direction. Then $\xi^{\perp}$ is almost umbilic.

Proof. Let $\left(X_{1}, \ldots, X_{n}\right)$ be an orthonormal basis as in [4] and $X_{1}, X_{2}$ such that this basis diagonalizes $A_{\xi}$. Then we have

$$
A_{\xi}=\left[\begin{array}{llll}
\lambda_{1} & & & 0 \\
& \lambda & & \\
& & \lambda & \\
0 & & & \lambda
\end{array}\right], \quad A_{\xi}=\left[\begin{array}{llll}
a & c & & 0 \\
c & b & & \\
& & \mu & \\
0 & & & \mu
\end{array}\right] \text {. }
$$

Now we can use the characterizaiton of conformally flat manifolds in terms of the sectional curvatures given by Kulkarni [3], namely,

$$
K\left(X_{1}, X_{2}\right)+K\left(X_{3}, X_{4}\right)=K\left(X_{1}, X_{3}\right)+K\left(X_{2}, X_{4}\right)
$$

for any orthonormal vectors $X_{1}, X_{2}, X_{3}, X_{4} \in T_{x} M$ (where $K\left(X_{i}, X_{j}\right)$ is the sectional curvature of the plane $\left.\left\{X_{i}, X_{j}\right\}\right)$, together with the Gauss equation to get

$$
\mu^{2}-(a+b) \mu+\left(a b-c^{2}\right)=0
$$

which implies that $\mu$ is an eigenvalue of $A_{\xi^{\perp}}$.

In [2] the authors affirmed that the example (2.1) contradicts [5] (which, at that time, was a conjecture). But, we have shown that (2.1) contradicts their result.

3. Proof of the theorem. We will start with the necessary part of the theorem. We will denote by $\nabla$ and $\langle$,$\rangle the Riemannian connection$ and metric respectively, $\alpha$ will be the second fundamental form and $\nabla^{\perp}$ the normal connection.

Let $\xi_{1}$ and $\xi_{2}$ be the differentiable almost umbilic directions of $f$. Let $\left(X_{1}, \ldots, X_{n}\right)$ be an orthonormal frame which diagonalizes $A_{\xi_{1}}$. Then

We have:

$$
A_{\xi_{1}}=\left[\begin{array}{llll}
\lambda_{1} & & & 0 \\
& \lambda & & \\
& & \lambda & \\
0 & & & \lambda
\end{array}\right], \quad A_{\xi_{2}}=\left[\begin{array}{llll}
a & c & & 0 \\
c & b & & \\
& & \mu & \\
0 & & & \mu
\end{array}\right] .
$$

$$
\begin{aligned}
& \alpha\left(X_{i}, X_{j}\right)=\alpha\left(X_{1}, X_{i}\right)=\alpha\left(X_{2}, X_{i}\right)=0, \quad i, j \geq 3, i \neq j, \\
& \alpha\left(X_{1}, X_{2}\right)=c \xi_{2} .
\end{aligned}
$$


If $\lambda_{1}=\lambda, \xi_{1}$ is umbilic and then $R^{\perp}=0$. Thus, we may assume $\lambda_{1} \neq \lambda$. Writing the Codazzi equation for $X_{i}, X_{j}$ and $\xi_{1}(i, j \geq 3, i \neq j)$, we get

$$
X_{i}(\lambda)+\mu\left\langle\nabla_{X_{i}}^{\perp} \xi_{2}, \xi_{1}\right\rangle=(\lambda-\lambda)\left\langle X_{i}, \nabla_{X_{i}} X_{j}\right\rangle .
$$

Hence, if $\mu \neq 0$ we have

$$
\left\langle\nabla_{X_{i}}^{\perp} \xi_{2}, \xi_{1}\right\rangle=-X_{i}(\lambda) / \mu, \quad i \geq 3 .
$$

Similarly, we obtain

$$
\left\langle\nabla_{X_{i}}^{\perp} \xi_{2}, \xi_{1}\right\rangle=-X_{i}(\lambda) / b, \quad i \geq 3 .
$$

If $b=\mu$, Gauss and Kulkarni ((2.4)) equations imply $k_{1}=K\left(X_{1}, X_{i}\right)$ $=K\left(X_{1}, X_{2}\right), i \geq 3$, contradicting that $M$ is of type $(2, \varepsilon)$. Then $b \neq \mu$, and this implies

$$
X_{i}(\lambda)=0=\left\langle\nabla_{X}^{\perp} \xi_{2}, \xi_{1}\right\rangle, \quad i \geq 3 .
$$

Now, the Codazzi equation for $X_{1}, X_{2}, X_{i}(i \geq 3)$ and $\xi_{1}$ gives

$$
\begin{gathered}
\left\langle\nabla_{X_{1}} X_{2}, X_{i}\right\rangle \lambda+\left\langle\nabla_{X_{1}} X_{i}, X_{2}\right\rangle \lambda=\left\langle\nabla_{X_{2}} X_{1}, X_{i}\right\rangle \lambda+\left\langle\nabla_{X_{2}} X_{i}, X_{1}\right\rangle \lambda_{1} \\
=\left\langle\nabla_{X_{1}} X_{1}, X_{2}\right\rangle \lambda+\left\langle\nabla_{X_{1}} X_{2}, X_{1}\right\rangle \lambda_{1}
\end{gathered}
$$

and since $\lambda \neq \lambda_{1}$ we have

$$
\left\langle\nabla_{X_{2}} X_{i}, X_{1}\right\rangle=\left\langle\nabla_{X_{i}} X_{2}, X_{1}\right\rangle=0 .
$$

Consider the following differentiable distributions:

$$
\begin{array}{ll}
D_{1}=\operatorname{span}\left\{X_{1}, X_{3}, \ldots, X_{n}\right\}, & D_{2}=\operatorname{span}\left\{X_{2}, X_{3}, \ldots, X_{n}\right\}, \\
D=\operatorname{span}\left\{X_{3}, \ldots, X_{n}\right\}, & D^{\perp}=\operatorname{span}\left\{X_{1}, X_{2}\right\} .
\end{array}
$$

$D$ is obviously integrable because $X_{3}, \ldots, X_{n}$ are tangent to the geometric sphere. From (3.1), we conclude that $D_{2}$ is integrable and, if we prove that $\left\langle\nabla_{X_{1}} X_{i}, X_{2}\right\rangle=0$, we will have $D_{1}$ and $D^{\perp}$ integrable. For this we apply the Codazzi equation to $X_{2}, X_{1}, X_{i},(i \geq 3)$, and $\xi_{2}$ to get

$$
\left\langle\nabla_{X_{1}} X_{2}, X_{i}\right\rangle(\mu-b)=c\left(\left\langle\nabla_{X_{1}} X_{1}, X_{i}\right\rangle-\left\langle\nabla_{X_{2}} X_{2}, X_{i}\right\rangle\right) .
$$

Since $\mu \neq b, D_{1}$ and $D^{\perp}$ will be integrable if and only if $c=0\left(R^{\perp}=0\right)$ or $\left\langle\nabla_{X_{1}} X_{1}, X_{i}\right\rangle=\left\langle\nabla_{X_{2}} X_{2}, X_{i}\right\rangle$. Let us suppose $c \neq 0$ in an open set $U$. We are going to prove that $\left\langle\nabla_{X_{1}} X_{1}, X_{i}\right\rangle=\left\langle\nabla_{X_{2}} X_{2}, X_{i}\right\rangle$.

First, we will consider the case $\varepsilon=0$. Then $U$ is a flat manifold. The Codazzi equation for $X_{1}, X_{2}, X_{i}$ and $\xi_{2}$ implies

$$
\left\langle\nabla_{X_{1}} X_{1}, X_{i}\right\rangle c-\left\langle\nabla_{X_{1}} X_{i}, X_{2}\right\rangle b=X_{i}(c)=\left\langle\nabla_{X_{2}} X_{2}, X_{i}\right\rangle c .
$$


We claim that $X_{i}(c)=0$, which implies $\left\langle\nabla_{X_{2}} X_{2}, X_{i}\right\rangle=0$, for $i \geq 3$. In fact, we can take in $\Sigma_{2}$, the maximal leaf of the integrable distribution $D_{2}$, local coordinates $\left(x_{2}, \cdots, x_{n}\right)$ such that $\partial / \partial x_{i}=\beta_{i} X_{i}$, since $D$ is integrable. Because $\Sigma_{2}$ is flat we can choose these coordinates such that $\beta_{i}=\beta$ and $\partial \beta / \partial x_{i}=0$ for each $i=2, \cdots, n . \Sigma_{2}$ is immersed in $\mathbf{R}^{n+2}$ and $X_{1}$ is normal vector for $\Sigma_{2}$. Now we compute $X_{i}(c)$.

$$
c=\left\langle A_{\xi_{2}} X_{2}, X_{1}\right\rangle=-1 / \beta\left\langle\tilde{\nabla}_{\partial / \partial x_{2}} \xi_{2}, X_{1}\right\rangle
$$

where $\tilde{\nabla}$ denotes the connection in $\mathbf{R}^{n+2}$. Then

$$
\partial c / \partial x_{i}=-1 / \beta\left\{\left\langle\tilde{\nabla}_{\partial / \partial X_{i}} \nabla_{\partial / \partial x_{2}} \xi_{2}, X_{1}\right\rangle+\left\langle\tilde{\nabla}_{\partial / \partial x_{2}} \xi_{2}, \tilde{\nabla}_{\partial / \partial X_{i}} X_{1}\right\rangle\right\}
$$

But the last term is zero, because $\varepsilon=0$. Since

$$
\begin{aligned}
0 & =\left\langle\tilde{R}\left(\partial / \partial x_{2}, \partial / \partial x_{i}\right) \xi_{2}, X_{1}\right\rangle \\
& =\left\langle\tilde{\nabla}_{\partial / \partial x_{2}} \tilde{\nabla}_{\partial / \partial x_{1}} \xi_{2}, X_{1}\right\rangle-\left\langle\tilde{\nabla}_{\partial / \partial x_{1}} \tilde{\nabla}_{\partial / \partial x_{2}} \xi_{2}, X_{1}\right\rangle
\end{aligned}
$$

and again because $\varepsilon=0, \tilde{\nabla}_{\partial / \partial X_{l}} \xi_{2}=0$, implying in (3.2)

$$
\partial c / \partial x_{i}=\beta X_{i}(c)=0 .
$$

Now, considering the orthonormal frame $\left(V_{1}, V_{2}, X_{3}, \ldots, X_{n}\right)$ diagonalizing $A_{\xi_{2}}$ we have

$$
A_{\xi_{1}}=\left[\begin{array}{cccc}
x & z & & 0 \\
z & y & & \\
& & 0 & \\
0 & & & 0
\end{array}\right], \quad A_{\xi_{2}}=\left[\begin{array}{llll}
0 & & & 0 \\
& \mu_{1} & & \\
& & 0 & \\
0 & & & 0
\end{array}\right] .
$$

Similarly, we conclude for $i \geq 3$

$$
\begin{gathered}
\left\langle\nabla_{X_{i}} V_{1}, V_{2}\right\rangle=0, \quad\left\langle\nabla_{V_{1}} X_{i}, V_{2}\right\rangle=0 \quad \text { and } \\
X_{i}(z)=z\left\langle\nabla_{V_{1}} V_{1}, X_{i}\right\rangle=0 .
\end{gathered}
$$

Setting $V_{1}=\cos \theta X_{1}+\sin \theta X_{2}$ and $V_{2}=\sin \theta X_{1}-\cos \theta X_{2}$, because $\left\langle\nabla_{V_{1}} X_{i}, V_{2}\right\rangle=0$, a straightforward computation shows

$$
\left\langle\nabla_{V_{1}} V_{1}, X_{i}\right\rangle=\left\langle\nabla_{X_{1}} X_{1}, X_{i}\right\rangle \text { and }\left\langle\nabla_{V_{2}} V_{2}, X_{i}\right\rangle=\left\langle\nabla_{X_{2}} X_{2}, X_{i}\right\rangle \text {. }
$$

Thus, (3.3), (3.4) and (3.5) imply

$$
\left\langle\nabla_{X_{2}} X_{2}, X_{i}\right\rangle=\left\langle\nabla_{X_{1}} X_{1}, X_{i}\right\rangle=0, \quad i \geq 3 .
$$

Now, if $\varepsilon \neq 0$, we can take a conformal diffeomorphism $\Phi$ from $U$ to an open set $O$ of the Euclidean space. We will denote again by $\left(V_{1}, V_{2}, X_{3}, \ldots, X_{n}\right)$ the orthonormal basis which diagonalizes $A_{\xi_{2}}$. Let $\gamma_{1}$ and $\gamma_{2}$ be the integral curves of $d \Phi\left(X_{1}\right)$ and $d \Phi\left(V_{2}\right)$ respectively. 
We can immerse $O$ isometrically in $\mathbf{R}^{n+2}$ by composing a cylindrical immersion of $\mathbf{R}^{n}$ to $\mathbf{R}^{n+2}$ whose principal direction is $\gamma_{1}^{\prime}(t)$, with another cylindrical immersion of $\mathbf{R}^{n+1}$ to $\mathbf{R}^{n+2}$ with $\gamma_{2}^{\prime}(t)$ as principal direction. Then by (3.6), we have

$$
\left\langle\nabla_{d \Phi\left(X_{1}\right)} d \Phi\left(X_{1}\right), d \Phi\left(X_{i}\right)\right\rangle=\left\langle\nabla_{d \Phi\left(X_{2}\right)} d \Phi\left(X_{2}\right), d \Phi\left(X_{i}\right)\right\rangle .
$$

Since $\Phi$ is conformal, writing the Riemannian connection in terms of the Riemannian metric, we get

$$
\left\langle\nabla_{X_{1}} X_{1}, X_{i}\right\rangle=\left\langle\nabla_{X_{2}} X_{2}, X_{i}\right\rangle \text {. }
$$

This proves that the distributions $D_{1}$ and $D^{\perp}$ are integrable. Now Lemma (3.3) of [6] finishes the first part of the proof.

The second part of the proof follows from Theorem (1.12) of [6].

REMARK. If $M$ is of type $(1, \varepsilon)$ with $\varepsilon=1$, taking $\xi_{1}, \xi_{2}, X_{1}, \ldots, X_{n}$ as in the above theorem, we have

$$
A_{\xi_{1}}=\left[\begin{array}{llll}
\lambda_{1} & & & 0 \\
& \lambda & & \\
& & \lambda & \\
0 & & & \lambda
\end{array}\right], \quad A_{\xi_{2}}=\left[\begin{array}{llll}
a & c & & 0 \\
c & b & & \\
& & \mu & \\
0 & & & \mu
\end{array}\right]
$$

with $b=\mu$, because $M$ is of type $(1, \varepsilon)$. Now, the Gauss and Kulkarni equations imply $R^{\perp}=0$.

\section{REFERENCES}

[1] R. L. Bishop and S. I. Goldberg, On conformally flat spaces with commuting curvature and Ricci transformations, Canad. J. Math., XXIV (1972), 799-804.

[2] B. Y. Chen and T. Teng, Flatness of normal connections of totally quasiumbilical submanifolds and its applications, Soochow, J. Math. and Mat. Sci., 1 (1975), 9-16.

[3] R. S. Kulkarni, Curvature structures and conformal transformations, J. Differential Geom., 4 (1970), 425-451.

[4] J. D. Moore, Conformally flat submanifolds of Euclidean space, Math. Ann., 225 (1977), 89-97.

[5] J. D. Moore and J. M. Morvan, Sous-variétés conformement plates de codimension quatre, C. R. Acad. Sc. Paris, 287 (1978), 655-657.

[6] M. H. Noronha, Conformally flat immersions in codimension two, Geometriae Dedicata, 23 (1987), 115-130.

[7] H. Reckziegel, Completeness of curvature surfaces of an isometric immersion, $\mathbf{J}$. Differential Geom., 14 (1979), 7-20.

Received January 7, 1987. The author is partially supported by FAPESP-SP-BRASIL.

UNIVERSIDADE ESTADUAL DE CAMPINAS-IMECC

13081-CAMPINAS-SP-BRASIL 